\title{
OTIMIZAÇÃO DA REAÇÃo DE INTERESTERIFICAÇÃO QUíMICA DO ÓLEO DE PALMA
}

Renato Grimaldi*, Lireny Aparecida Guaraldo Gonçalves e Marlene Yumi Ando

Departamento de Tecnologia de Alimentos, Faculdade de Engenharia de Alimentos, Universidade Estadual de Campinas, CP 6091, 13081-970 Campinas - SP

Recebido em 28/5/04; aceito em 14/10/04; publicado na web em 17/2/05

\begin{abstract}
OPTIMIZATION OF THE CEMICAL INTERESTERIFICATION REACTION OF PALM OIL. The Brazilian market has been showing a growing concern with nutritional values of oil components of foods. Chemical interesterification is a promising alternative to the current processes of modifying the consistency of oils. Chemical interesterification of deodorized palm oil was studied on a laboratory scale. The best results were obtained with $0.4 \% \mathrm{MeONa}$ and heating for $20 \mathrm{~min}$ at $100{ }^{\circ} \mathrm{C}$. These conditions are based on the largest variation in triacylglycerols as compared to a control. The trisaturated values varied from 6.2 to $9.9 \%$, showing that the consistency of the oil improved for it to be used in margarines, without the formation of trans isomers.
\end{abstract}

Keywords: palm oil; chemical interesterification; solid fat content.

\section{INTRODUÇÃO}

A interesterificação química oferece uma importante alternativa para modificar o comportamento de óleos e gorduras, sem alterar os ácidos graxos do material de partida. As alterações nas propriedades de solidificação e fusão dos óleos e gorduras interesterificadas são ocasionadas pelas mudanças relativas dos componentes glicerídeos, após o rearranjo dos ácidos $\operatorname{graxos}^{1,2}$.

No caso específico do óleo de palma, a interesterificação química produz aumento no ponto de gotejamento como resposta ao maior teor de triacilgliceróis trisaturados. Além disso, ocorre aumento da sua plasticidade, caracterizado pelo alto teor de sólidos à temperatura ambiente ${ }^{3,4}$. Para o óleo de palma, que apresenta problema de pós-endurecimento e de cristalização lenta, a reação de interesterificação pode diminuir em muito este efeito ${ }^{5}$.

Um importante parâmetro na avaliação do produto interesterificado é a composição triacilglicerídica, determinante na caracterização de mudanças físicas e na forma cristalina do produto formulado. A técnica de cromatografia líquida de alta eficiência é uma ferramenta de fácil manuseio, onde a dificuldade fica por conta da identificação dos picos, uma vez que a separação é feita pelos compostos individuais. As outras técnicas cromatográficas que podem ser utilizadas na quantificação dos triacilglicerídios presentes são a cromatografia gasosa com coluna curta empacotada e a cromatografia gasosa de alta resolução a altas temperaturas com colunas apolares. Nesses dois casos, a identificação dos triacilglicerídios é limitada, pois é baseada no número de átomos de carbono presentes.

Este trabalho teve como objetivo otimizar as condições da reação de interesterificação química em pequena escala, utilizando para isto óleo de palma desodorizado.

\section{PARTE EXPERIMENTAL}

\section{Material}

Óleo de palma desodorizado, fornecido pela CRA (Companhia Refinadora da Amazônia) e obtido por refino físico.

Metóxido de sódio (100\%) em pó anidro (BASF).

*e-mail: grimaldi@fea.unicamp.br

\section{Métodos}

Planejamento experimental

Otimização da reação de interesterificação química - realizada através de um experimento fatorial completo.

Fatores: concentração de catalisador (3 níveis - 0,2, 0,3 e 0,4\%); tempo de reação (3 níveis - 20, 40 e $60 \mathrm{~min}$ ); temperatura $-100{ }^{\circ} \mathrm{C}$.

No caso da variável temperatura, optou-se pelo valor intermediário normalmente usado $\left(90-110{ }^{\circ} \mathrm{C}\right)$, uma vez que o número de experimentos seria muito superior e que, intrinsecamente, a variável tempo poderia ajustar a cinética da reação.

Total de experimentos $-3 \times 3 \times 2$ (repetição) $=18$, escolhidos aleatoriamente.

Resposta: níveis de diferentes triacilglicerídeos obtidos por CLAE.

Avaliação dos dados: através do Programa Statística versão 5.0, com diagrama de caixas e teste comparativo entre o controle e as reações através do teste de Dunnett com $5 \%$ de significância ${ }^{6}$.

Os tratamentos foram identificados conforme Tabela 1.

Reações de Interesterificação Química - Escala laboratorial de 100 g. com agitação magnética.

Metodologias analíticas - conforme métodos oficiais da $\mathrm{AOCS}^{7}$. Composição em ácidos graxos - Método AOCS Ce 1f-96 (97). O preparo dos ésteres metílicos foi através do método AOCS Ce 2-66 (97). As condições de operação do cromatógrafo foram: Cromatógrafo Gasoso Capilar CGC Agilent 6850 Series GC System, dotado de coluna capilar DB-23 AGILENT(50\% cyanopropyl)methylpoysiloxane, dimensões $60 \mathrm{~m}, \phi$ int: $0,25 \mathrm{~mm}, 0,25 \mu \mathrm{m}$ filme. Temperatura do forno $-195{ }^{\circ} \mathrm{C}-20 \mathrm{~min}, 195-215^{\circ} \mathrm{C}\left(5^{\circ} \mathrm{C} /\right.$ $\min ), 215{ }^{\circ} \mathrm{C}-16 \mathrm{~min}$; temperatura do detector: $280{ }^{\circ} \mathrm{C}$; temperatura do injetor: $250{ }^{\circ} \mathrm{C}$; gás de arraste: Hélio; split: 1:50.

Conteúdo de gordura sólida - Método AOCS Cb 16b-93 (97). Método direto, temperagem para gorduras não estabilizadas, leituras das amostras em série, nas temperaturas de 10, 20, 25, 30, 35, 40 e $45{ }^{\circ} \mathrm{C}$. Equipamento - Espectrômetro de ressonância magnética nuclear Bruker pc120.

Ácidos graxos livres - Método AOCS Ca 5a-40 (97)

Umidade e matéria volátil - Método AOCS Ca 2c-25 (97)

Índice de peróxido - Método AOCS Cd 8-53 (97)

Sabões - Método AOCS Cc 17-79 (97) 
Tabela 1. Identificação dos tratamentos utilizados na otimização da interesterificação química do óleo de palma

\begin{tabular}{|c|c|c|c|c|c|c|c|c|c|c|}
\hline & \multicolumn{10}{|c|}{ Tratamentos $-100^{\circ} \mathrm{C}$} \\
\hline & 1 & 2 & 3 & 4 & 5 & 6 & 7 & 8 & 9 & 10 \\
\hline$\% \mathrm{MeONa}$ & 0,2 & 0,2 & 0,2 & 0,3 & 0,3 & 0,3 & 0,4 & 0,4 & 0,4 & 0 \\
\hline Tempo de reação (min) & 20 & 40 & 60 & 20 & 40 & 60 & 20 & 40 & 60 & 0 \\
\hline
\end{tabular}

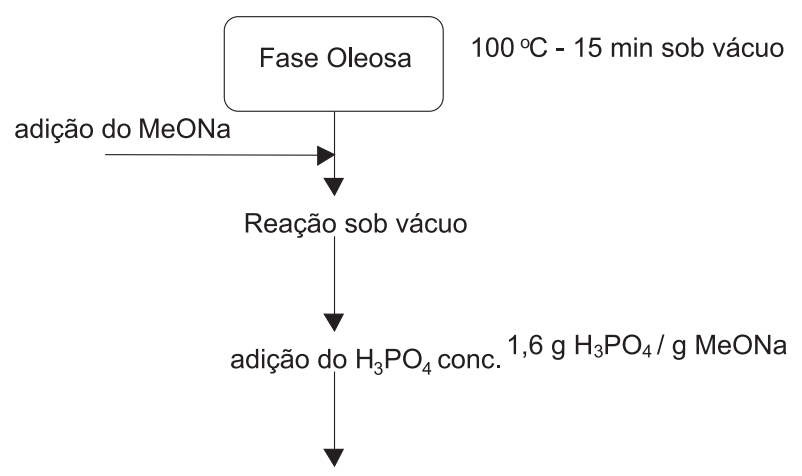

3 lavagens com $10 \%$ de $\mathrm{H}_{2} \mathrm{O}$ quente $\left(90^{\circ} \mathrm{C}\right)$

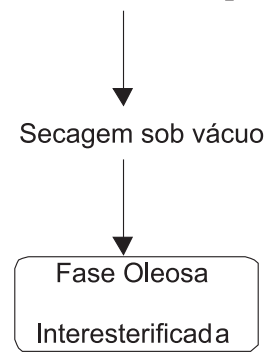

Figura 1. Esquema da reação de interesterificação química

Composição triacilglicerídica (CLAE - cromatografia líquida de alta eficiência): solução $5 \%$ em acetona, Cromatógrafo Líquido Perkin Elmer 250; coluna Lichrosorb RP-18 de $25 \mathrm{~cm} \mathrm{x} \mathrm{4,6} \mathrm{mm} \mathrm{x}$ $5 \mu \mathrm{m}$ Merck; fase móvel - acetona:acetonitrila (62:38); fluxo $1 \mathrm{~mL} / \mathrm{min}$; Detector de Índice de Refração Sicon Analytic; Integrador: HP 3395.

\section{RESULTADOS E DISCUSSÃO}

A qualidade do óleo utilizado na reação de interesterificação química é fundamental. "Venenos" catalíticos podem provocar queda na atividade do metóxido de sódio.

Essa qualidade está relacionada com os teores de ácidos graxos livres, índice de peróxido, umidade e sabões ${ }^{8}$. O óleo de palma original não apresentou residual de sabões pelo fato de ter sido obtido através do refino físico. A Tabela 2 mostra as características apresentadas pela matéria-prima utilizada nesta otimização.

A qualidade do óleo inicial, aqui chamado de controle, é fundamental para a reação de interesterificação química. A Tabela 3 mostra a composição em ácidos graxos do óleo de palma.

Interesterificação química é um processo de difícil controle. Muitos autores citam condições de reação, mas a prática diz que os mesmos são inerentes aos equipamentos e material utilizados. Rousseau et $a l .{ }^{9}$ realizaram otimização do tempo da reação de interesterificação, com lotes de 100 g e 0,5\% de metóxido de sódio. A variabilidade das condições da reação de interesterificação química fica evidente em outras citações, onde se encontram valores médios de $0,5 \%$ de metóxido de sódio, mas temperaturas entre
Tabela 2. Caracterização da matéria-prima

\begin{tabular}{lc}
\hline Característica & Valor \\
\hline Umidade $(\%)$ & 0,04 \\
AGL $(\%$ como ác. palmítico) & 0,08 \\
Índice de peróxido (meq $\mathrm{O}_{2} / \mathrm{kg}$ amostra) & 0 \\
Sabões $(\mathrm{mg} / \mathrm{kg})$ & Isento \\
\hline
\end{tabular}

Tabela 3. Composição em ácidos graxos (\%) do óleo de palma

\begin{tabular}{lc}
\hline Ácido Graxo & $\%(\mathrm{~m} / \mathrm{m})$ \\
\hline C 12:0 - Láurico & 1,1 \\
C 14:0 - Mirístico & 1,0 \\
C 16:0 - Palmítico & 40,7 \\
C 18:0 - Esteárico & 5,0 \\
C 18:1 - Oléico & 42,0 \\
C 18:2 - Linoléico & 10,2 \\
\hline Saturados & 47,8 \\
Insaturados & 52,2 \\
\hline
\end{tabular}

$70{ }^{\circ} \mathrm{C}$ e tempo de reação de $1 \mathrm{~h}$ e $95{ }^{\circ} \mathrm{C}$ com 10 min de reação. Além disso, a interesterificação química promove um rearranjo randômico dos ácidos graxos na molécula do glicerol ${ }^{10,11}$.

Neste trabalho, optou-se por uma otimização da reação de interesterificação química, pois testes iniciais com $0,2 \%$ de catalisador, valor também citado na literatura, não apresentaram bons resultados. As Figuras 2, 3 e 4 mostram o perfil de sólidos das amostras nas condições especificadas no planejamento experimental, sempre em relação ao controle. Esses resultados representam o perfil médio de triplicata da reação.

A confirmação da reação de interesterificação é freqüentemente monitorada pela alteração da cor, ponto de fusão e perfil de sólidos. Segundo vários autores o desenvolvimento da cor marrom não é suficiente para confirmar o rearranjo completo, afetado principalmen-

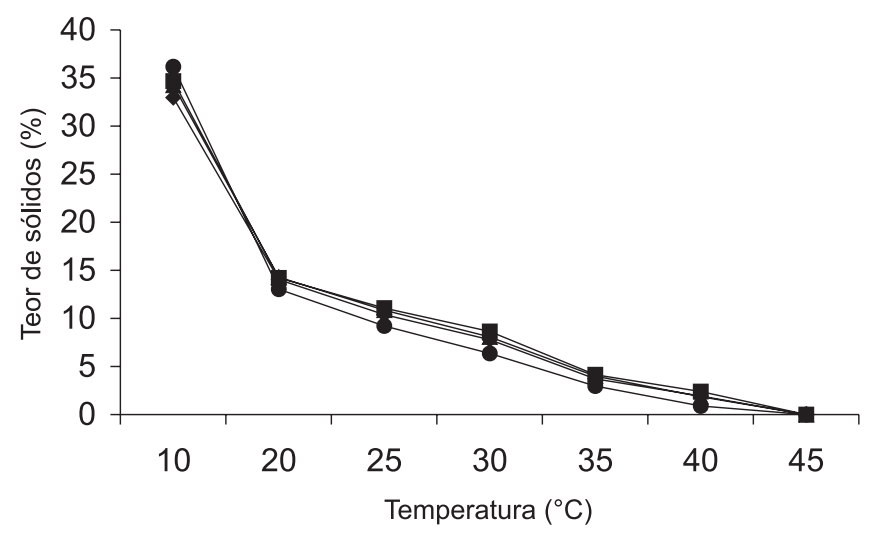

Figura 2. Curvas de sólidos em amostras de óleos de palma interesterificados (0,2\% MeONa). - - PO-controle; - - 0,2\%, 20'; - $\mathbf{-}-0,2 \%, 40$; 0,2\%, 60 ' 
te pelos parâmetros qualidade e teor do catalisador, temperatura de reação e qualidade do óleo ${ }^{1,3,12}$. A escolha da melhor condição, em alguns trabalhos, foi baseada no menor tempo de reação necessário para obtenção da máxima diferença do perfil de sólidos? .

No presente trabalho, determinou-se a composição triacilglicerídica das amostras, através da cromatografia líquida de alta eficiência, com a identificação dos picos individuais ${ }^{13}$. Os resultados médios da composição triacilglicerídica dos 9 tratamentos e do óleo de palma controle são mostrados na Tabela 4.

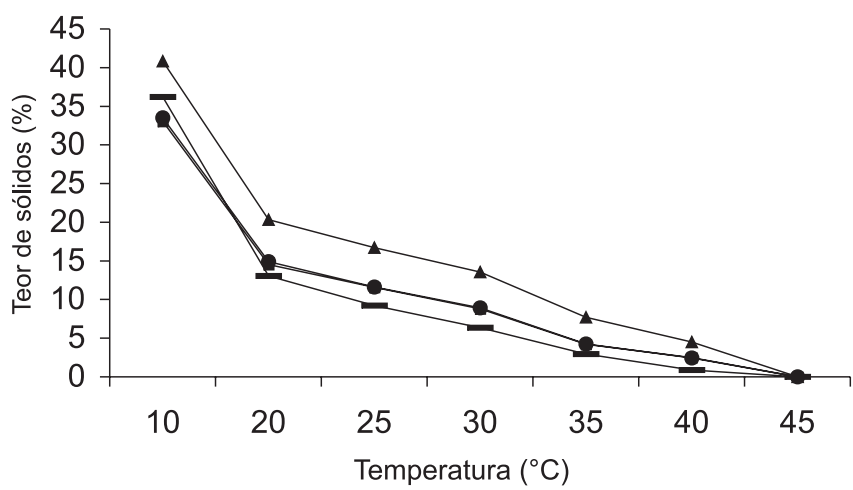

Figura 3. Curva de sólidos em amostras de óleos de palma interesterificados $(0,3 \% \mathrm{MeONa}) .=-$ PO-controle; - - 0,3\%, 20'; - $-0,3 \%, 40^{\prime} ;-\mathbf{\Delta}-$ $0,3 \%, 60^{\prime}$

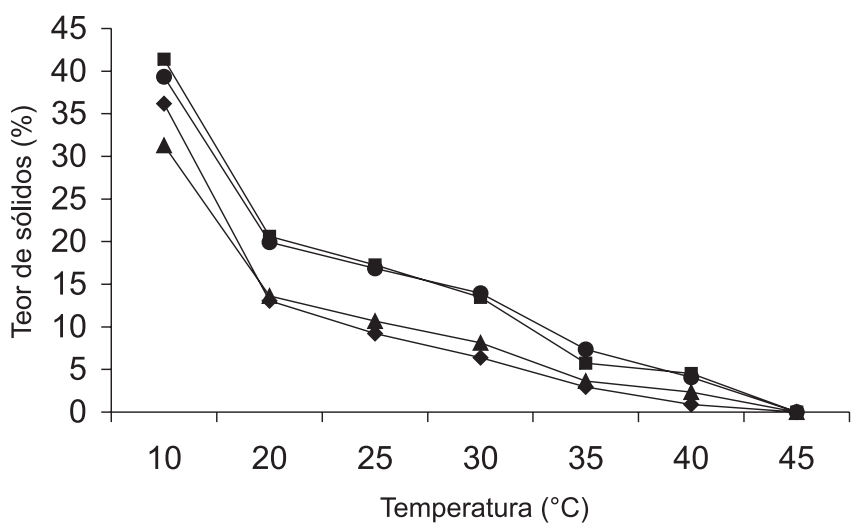

Figura 4. Curva de sólidos em amostras de óleos de palma interesterificados $(0,4 \% \mathrm{MeONa}) .->-P O$-controle; - $\mathbf{-}-0,4 \%, 20^{\prime} ;-\mathbf{0}-0,4 \%, 40^{\prime} ;-\mathbf{\Delta}-$ $0,4 \%, 60$ '
Uma ferramenta bastante útil na avaliação do desempenho da interesterificação é a cromatografia de triacilgliceróis, quer seja obtida por cromatografia líquida de alta eficiência, quer seja por cromatografia gasosa a alta temperatura. Um exemplo prático do uso desta técnica pode ser citado como a atenção centralizada, no grupo C54, onde através de quantificações dos triacilglicerideos SSS e SOS, na ocorrência do "fat bloom", a proporção SOS:SSS se altera de 1:1, característica da manteiga de cacau, para valores próximos de $8: 1^{14}$. Outras proporções podem ser avaliadas para se relacionar alterações de gorduras em produtos processados, que corroborem com indicações práticas de processamento incorreto ou incompatibilidade de gorduras como, por exemplo, no caso de substitutos ou equivalentes de manteiga de cacau. A alteração da composição triacilglicerídica através da interesterificação química modifica as propriedades físicas e o comportamento de cristalização do óleo, tornando-se uma boa alternativa ao processo de hidrogenação na produção de gorduras plásticas utilizadas na fabricação de margarinas ${ }^{15}$. Porém, pelo fato da alteração ser randômica, há limitação da amplitude da aplicação quando comparada ao processo de hidrogenação ${ }^{16}$.

Óleo de palma apresenta triacilglicerídeos de alto ponto de fusão como PPP (PF 65, ${ }^{\circ} \mathrm{C}$ ), mas se funde na faixa de $37-42{ }^{\circ} \mathrm{C}$ com 3-4\% de sólidos. Em sua composição detectam-se triacilglicerídeos que não são simples como o PPP, mas misturas deste com outros acilgliceróis mistos, de pontos de fusão intermediários como POP e $\mathrm{PPO}^{17}$

Na Figura 5 é mostrado o perfil cromatográfico da composição triacilglicerídica obtida por CLAE do óleo de palma antes da interesterificação.

Fez-se uma avaliação estatística dos teores de SSS (trisaturados), UUU (tri-insaturados), SSU (monoinsaturados) e SUU (di-insaturados) através do Programa Statistica (versão 5.0), com comparação dos resultados obtidos através do teste de Dunnett, com $5 \%$ de significância ${ }^{6}$. A Figura 6 mostra os diagramas de caixas contendo os valores de SSS e UUU, com a identificação dos tratamentos.

Os dados de UUU e SSS, em nível de $0,2 \%$ de catalisador (tratamentos 1,2 e 3 ) comparados ao tratamento 10 (controle), praticamente não apresentam diferenças confirmadas pelos dados obtidos nas curvas de sólidos das amostras interesterificadas (Figura 2), evidenciando ausência de catalisador disponível para efetivar a transferência interna dos ácidos graxos na molécula.

O teor de catalisador de $0,3 \%$ indica variação média dos triacilgliceróis em relação a $0,2 \%$, porém uma alteração brusca

Tabela 4. Composição triacilglicerídica $(\%)$ de amostras de óleo palma original e interesterificadas a $100{ }^{\circ} \mathrm{C}$

\begin{tabular}{|c|c|c|c|c|c|c|c|c|c|c|}
\hline \multirow[b]{2}{*}{ TG } & \multicolumn{10}{|c|}{ Tratamentos } \\
\hline & 1 & 2 & 3 & 4 & 5 & 6 & 7 & 8 & 9 & 10 \\
\hline [cat ] & 0,2 & 0,2 & 0,2 & 0,3 & 0,3 & 0,3 & 0,4 & 0,4 & 0,4 & 0 \\
\hline tempo & 20 & 40 & 60 & 20 & 40 & 60 & 20 & 40 & 60 & 0 \\
\hline OLL & 0,6 & 0,5 & 0,6 & 0,6 & 0,6 & 0,9 & 1,0 & 0,9 & 0,5 & 0,4 \\
\hline PLL & 2,8 & 2,0 & 3,0 & 2,0 & 2,5 & 1,3 & 1,8 & 1,7 & 1,9 & 3,0 \\
\hline OLO & 2,6 & 3,2 & 3,5 & 2,7 & 2,7 & 5,1 & 5,0 & 5,0 & 2,9 & 2,6 \\
\hline PLO & 10,9 & 10,5 & 11,7 & 10,0 & 10,5 & 11,5 & 11,1 & 11,4 & 11,0 & 11,1 \\
\hline PLP & 7,6 & 7,0 & 8,5 & 6,5 & 7,9 & 7,2 & 6,1 & 6,5 & 7,3 & 8,8 \\
\hline OOO & 5,7 & 6,20 & 5,9 & 6,0 & 6,5 & 8,2 & 7,5 & 8,0 & 6,3 & 5,6 \\
\hline POO & 26,9 & 26,0 & 24,8 & 26,0 & 26,0 & 23,9 & 24,0 & 24,0 & 27,2 & 26,6 \\
\hline POP & 28,0 & 26,6 & 24,8 & 27,6 & 26,8 & 23,8 & 24,7 & 24,2 & 27,6 & 27,5 \\
\hline PPP & 5,0 & 5,5 & 5,0 & 6,9 & 5,4 & 6,9 & 7,1 & 7,0 & 5,5 & 4,7 \\
\hline SOO & 3,0 & 4,0 & 4,3 & 3,8 & 2,7 & 2,1 & 3,2 & 2,0 & 2,6 & 2,8 \\
\hline POS & 5,5 & 5,9 & 6,4 & 6,0 & 5,7 & 5,4 & 5,8 & 5,5 & 5,5 & 5,4 \\
\hline PPS & 1,6 & 1,5 & 1,6 & 1,9 & 2,8 & 3,8 & 2,8 & 3,8 & 1,8 & 1,5 \\
\hline
\end{tabular}




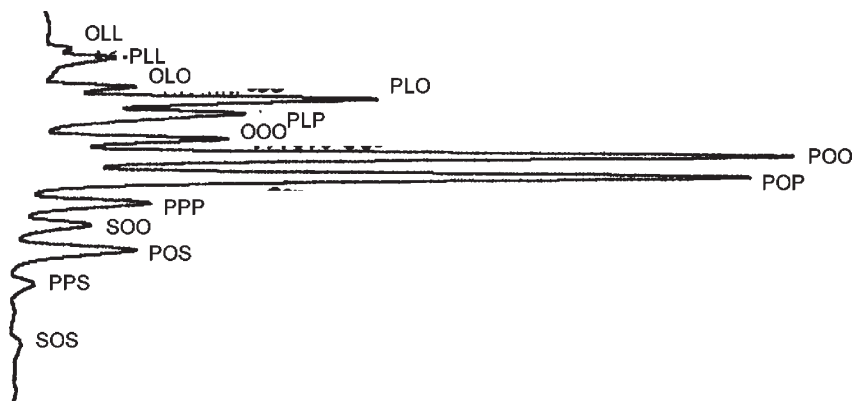

Figura 5. Perfil cromatográfico (CLAE) da composição triacilglicerídica do óleo de palma antes da interesterificação
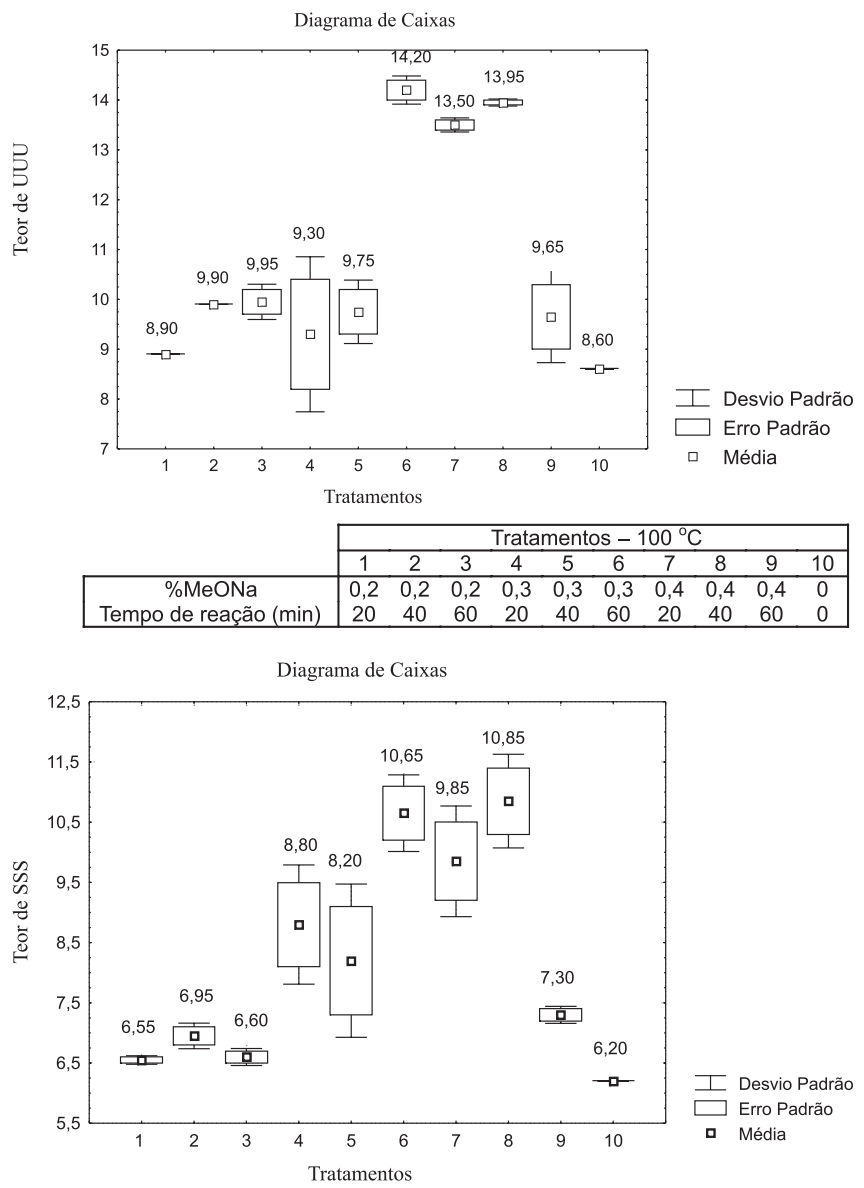

Figura 6. Diagrama de caixas para valores de UUU e SSS sob diferentes tratamentos

quando a reação é levada a efeito durante muito tempo (60 min), conforme dado da Figura 3. A curva de sólidos obtida para $60 \mathrm{~min}$ mostra produtos de maior consistência.
Todos os dados obtidos com reações realizadas com $0,4 \%$ de $\mathrm{MeONa}$ (Figura 4) apresentam o mesmo perfil triacilglicerídico com tempos de 20 e $40 \mathrm{~min}$. Os teores de triacilglicerídeos analisados foram os que mais se distanciaram da amostra inicial, evidenciando as trocas internas de ácidos graxos nas moléculas. No entanto, a 60 min observa-se teores de triacilglicerídeos praticamente idênticos aos observados com $0,2 \%$ de catalisador, evidência esta não confirmada de que a um tempo excessivamente longo talvez haja oportunidade de ocorrer um rearranjo contínuo. Estes rearranjos seriam favorecidos pelo excesso de catalisador, pois com 0,2 e $0,3 \%$ não foram observadas estas ocorrências.

Apenas os tratamentos 6,7 e $8(0,3 / 60,0,4 / 20$ e 0,4/40) apresentaram diferenças significativas a nível de $5 \%$ com relação ao controle, segundo o método empregado ${ }^{7}$.

A variabilidade nos teores de triacilglicerídeos em relação ao controle em conjunto com o menor tempo de reação foi o fator determinante na escolha da melhor condição. A partir dos resultados estatísticos, optou-se pela condição $0,4 \%$ de $\mathrm{MeONa}$ e $20 \mathrm{~min}$ de reação.

\section{CONCLUSÃO}

A reação de interesterificação química, apesar de bastante divulgada em termos de parâmetros de processo, deve ser sempre otimizada sob as condições do trabalho. Fatores como qualidade da matéria prima e catalisador, agitação, temperatura e tempo de reação são passíveis de interferências na qualidade do óleo final. Nesse trabalho, a condição escolhida como ótima foi com $0,4 \%$ de $\mathrm{MeONa}$ e 20 min de reação.

A interesterificação é uma ferramenta fundamental para o desenvolvimento de produtos gordurosos, de alta plasticidade e sem a presença dos ácidos graxos trans.

\section{REFERÊNCIAS}

1. Erickson, D. R.; Practical Handbook of Soybean Processing and Utilization, AOCS Press, Champaign: Illinois, 1995.

2. Castro, H. F.; Mendes, A. A.; Santos, J. C.; Quim. Nova 2004, 27, 146.

3. Laning, S. J.; J. Am. Oil Chem. Soc. 1985, 62, 400.

4. Duns, M. L.; J. Am. Oil Chem. Soc. 1985, 62, 408.

5. Timms, R. E.; Fette Seifen. Anstrichmittel. 1986, 8, 294.

6. Montgomery, D. C.; Design and Analysis of Experiments, $2^{\text {nd }}$ ed., New York, 1984.

7. AOCS; Official Methods and Recommended Practices of the American Oil Chemist's Society, Champaign: Illinois, $5^{\text {th }}$ ed., 1998.

8. Rozendaal, A.; INFORM. 1992, 3, 1232.

9. Rousseau, D.; Forestière, K.; Hill, A. R.; Marangoni, A. G.; J. Am. Oil Chem. Soc. 1996, 73, 963.

10. Tan, C. P.; Che Man, Y. B.; Food Chem. 2002, 76, 89.

11. Rodrigues, J. N.; Gioielli, L. A.; Food Res. Int. 2003, 36, 149.

12. Gioielli, L. A.; Baruffaldi, R.; Rev. Farm. e Bioquím. Univ. S. Paulo 1987, $24,29$.

13. Deffense, E.; Revue Française des Corps Gras 1984, 31, 123.

14. Traitler, H.; Dieffenbacher, A.; J. Am. Oil Chem. Soc. 1985, 62, 417.

15. Alpaslan, M.; Karaali, A.; Food Chem. 1998, 61, 301.

16. Ghotra, B. S.; Dyal, S. D.; Narine, S. S.; Food Res. Int. 2002, 35, 1015.

17. Kheiri, M. S. A.; J. Am. Oil Chem. Soc. 1985, 62, 410. 